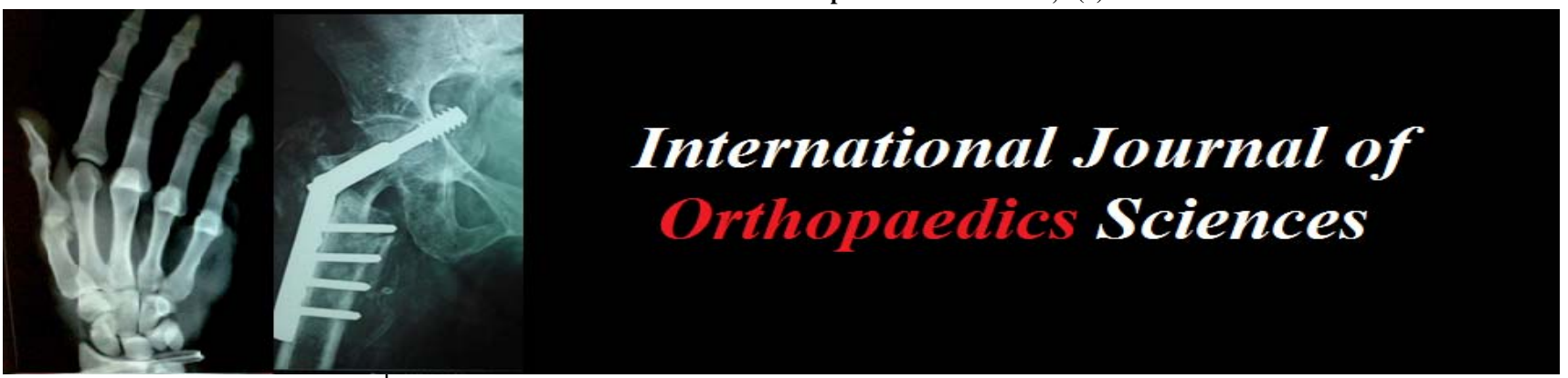

ISSN: $2395-1958$

IJOS 2017; 3(3): 253-255

(C) 2017 IJOS

www.orthopaper.com

Received: 25-05-2017

Accepted: 26-06-2017

Dr. Agni Raj R

Assistant Professor, Department

of Orthopedics, Government

Stanley Medical College,

Tamil Nadu, India

Dr. Tholgapiyan T

Professor, Department of

Orthopedics, Government

Stanley Medical College,

Tamil Nadu, India

Dr. Kathir Azhagan S

Resident Department of

Orthopedics, Government

Stanley Medical College,

Tamil Nadu, India

Dr. Mohan Kumar K

Professor, Department of

Orthopedics, Government

Stanley Medical College,

Tamil Nadu, India
Correspondence

Dr. Tholgapiyan T

Professor, Department of

Orthopedics, Government

Stanley Medical College,

Tamil Nadu, India

\section{Anterior cervical discectomy and fusion in patients with cervical disc disease- a clinical outcome study}

\author{
Dr. Agni Raj R, Dr. Tholgapiyan T, Dr. Kathir Azhagan S and Dr. Mohan \\ Kumar K
}

DOI: http://dx.doi.org/10.22271/ortho.2017.v3.i3d.50

Abstract

Introduction: Cervical disc disease is a common and distressing problem. For patients who do not respond to conservative treatment, surgical treatment in the form of cervical discectomy and fusion (ACDF) is an option. ACDF is challenging with respect to both patient selection and choice of surgical procedure. The aim of this study is to evaluate the clinical outcome of ACDF in patients with cervical disc disease.

Materials and methods: This study was conducted over a period of five years from 2008 to 2013 . The study group included eight male patients and four female patients. Maximum age of the patient was sixty years and minimum age was twenty six years. Maximum period of follow up was four years and minimum was six months. All patients underwent anterior cervical discectomy and fusion with autologous iliac bone graft with instrumentation (2 patients) or without instrumentation (10 patients). For all patients postoperative period was uneventful.

Results: Clinical outcome was measured using Visual Analogue Scale (VAS) and Cervical Oswestry pain disability score before and after surgery. Based on VAS, all patients had severe pain before surgery and at follow up ten patients had little or no pain and two patients had moderate pain. Based on Cervical Oswestry score, ten patients showed good score, one had fair and one had poor score.

Conclusion: ACDF is an effective treatment for cervical disc disease in patients who failed adequate period of conservative treatment.

Keywords: patients, Cervical disc, conservative treatment, surgical treatment, ACDF

\section{Introduction}

Cervical Disc Disease (CDD) is a common problem in the young adult population. It is slightly more common in men. Jobs which entails lifting of heavy weights, vehicle driving and cigarette smoking are associated with a higher incidence of cervical disc disease ${ }^{[1]}$. The basic pathophysiology of CDD is physiological changes in nucleus followed by progressive annular degeneration. Hypertrophic changes eventually develop around the facet joints and vertebral bodies $^{1}$. Patient complaints of neck pain, medial scapular pain and shoulder pain. Cranial symptoms like headache and vertigo may be seen. Symptoms of root compression associated with pain radiating to arm or chest with numbness in the fingers and motor weakness may be present. Radiographic evaluation of the cervical spine frequently shows loss of normal cervical lordosis and disc space narrowing. MRI of the cervical spine confirms the diagnosis. The vast majority of patients with CDD respond well to conservative treatment ${ }^{2}$. For those who do not respond to conservative treatment, surgical treatment in the form of anterior cervical discectomy and fusion (ACDF) is an option ${ }^{[3,4]}$. The other available options are anterior cervical discectomy alone and cervical arthroplasty.

\section{Aim of the study}

The aim of this study is to evaluate the clinical outcome of ACDF in twelve patients with cervical disc disease in whom conservative management had failed.

\section{Materials and methods}

This is a retrospective single-centre study of patients who underwent ACDF for Cervical disc disease. The study was performed over a period of 5 years from 2008 to 2013. 
The study group included eight males and four female patients. Maximum age of the patient was 60 years and minimum age was 26 years. Patients with persistent severe neck pain not responding to conservative treatment for over three months, Cervical radiculopathy with progressive paresis and MRI documented CDD with compression of nerve roots that correlates well with the clinical symptoms and signs were included in the study. The exclusion criteria included patients with cervical trauma, cervical neoplasia or ongoing cervical spine infection. All patients were subjected to a detailed clinical and neurological examination followed by x-ray and MRI evaluation of the cervical spine.

All patients underwent anterior cervical discectomy and fusion with autologous iliac bone graft with instrumentation (Locking cervical plate) in two patients and without instrumentation in ten patients. A right sided anterior approach to the cervical spine was used to expose the site of pathology. The appropriate cervical disc space was identified using image intensifier and cervical discectomy was performed at one or two levels based on the existing pathology. Two level discectomy was done in four patients. Bilateral nerve root decompression was ensured in all patients. Fusion was attained with autologous tricortical iliac bone graft. Instrumentation in the form of locking cervical plate was used in only two patients where two level discectomy had been performed. Wound drainage was not routinely used. Mean duration of the surgery was one to one and a half hours. There were no intraoperative complications. Postoperative period was uneventful.

\section{Results}

Clinical outcome was measured using Visual Analogue Scale (VAS) and Cervical Oswestry pain disability score both before and after surgery. Maximum period of follow up was four years and minimum period was six months. The following parameters were registered at follow up: neck pain, headache, lifting weight, driving, working status, sleeping and patient satisfaction with the surgical treatment. The following results were noticed. Based on VAS, all 12 patients had severe pain before surgery and 10 patients had no or mild pain at follow up and 2 patients had moderate pain at follow up. Based on Cervical Oswestry Neck Disability Index, 10 patients (84\%) showed good score, 1 patient (8\%) showed fair and 1 patient (8\%) showed poor score.
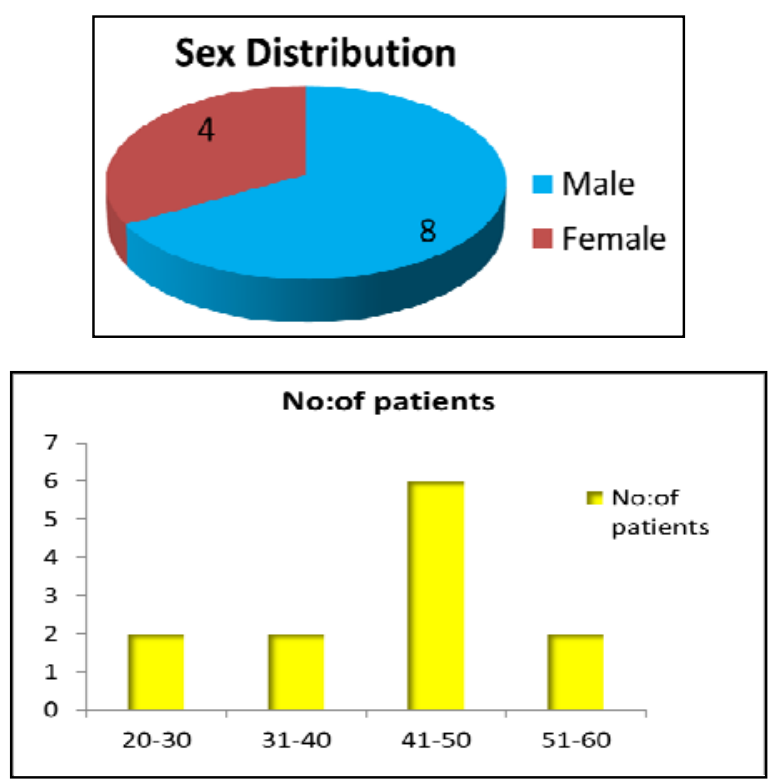
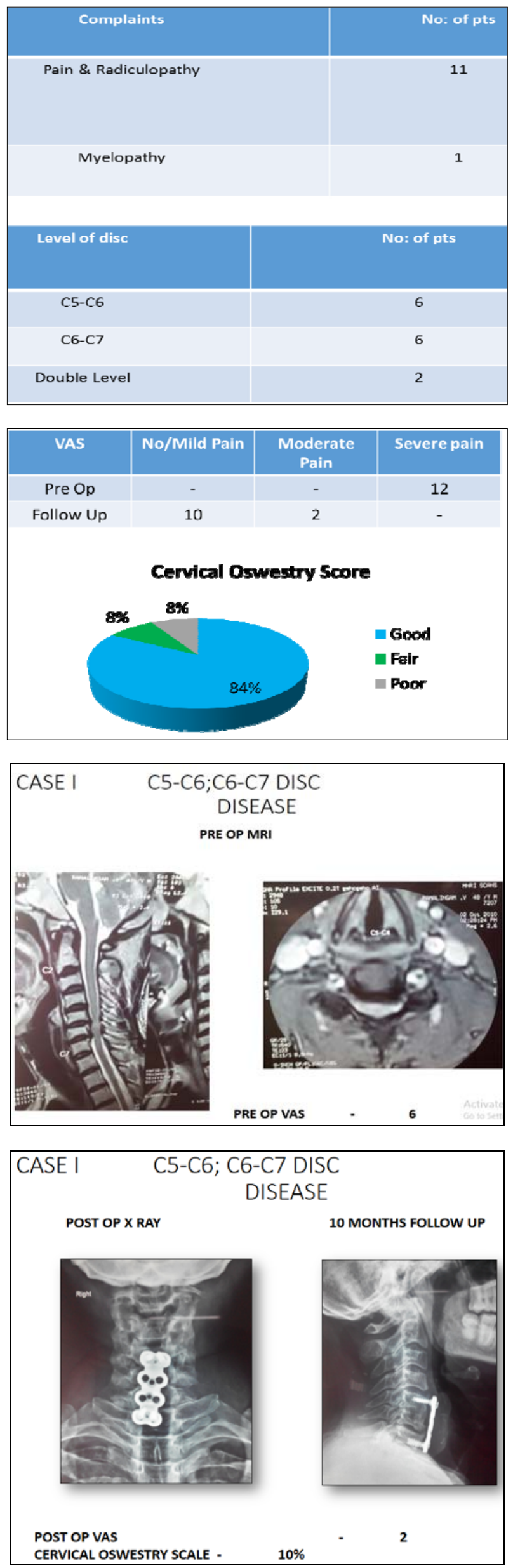

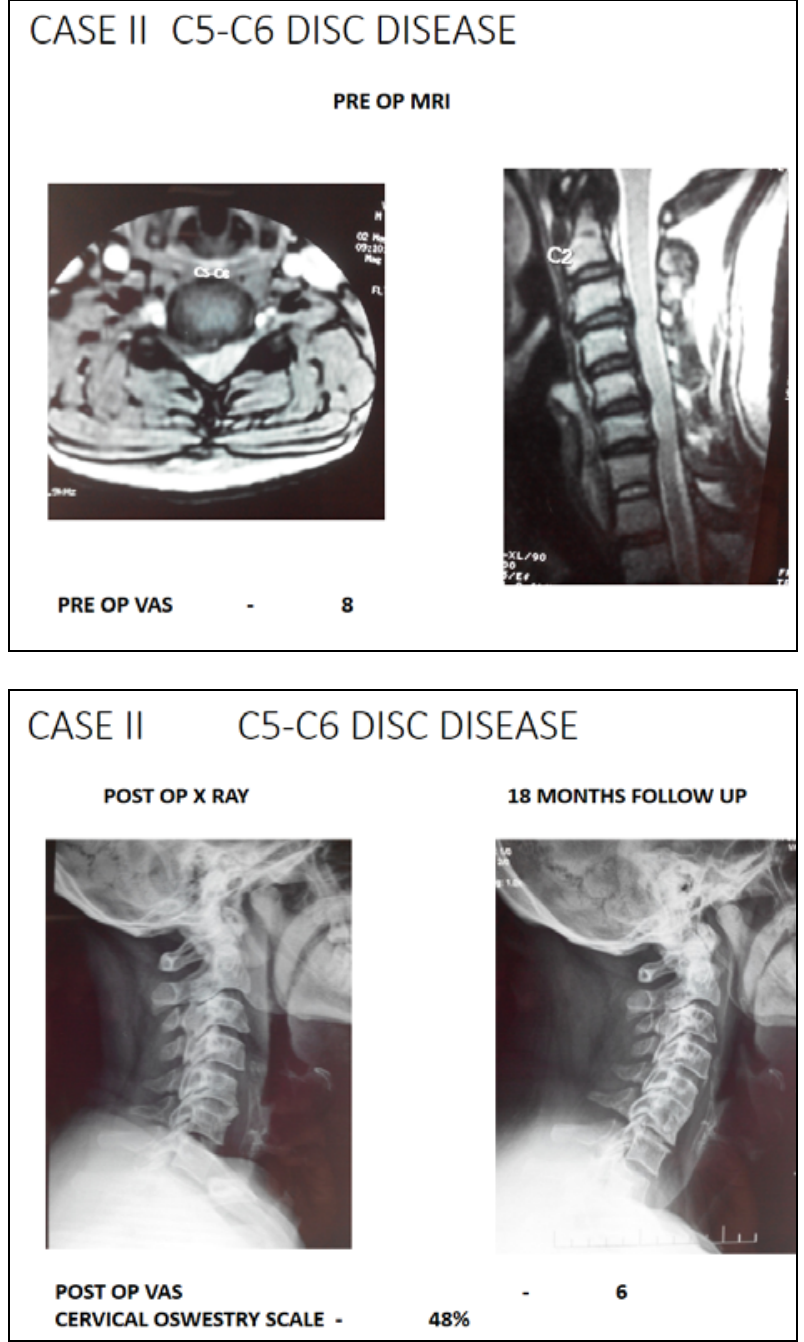

\section{Discussion}

The primary indications for operative treatment for cervical disc disease are 1) failure of nonoperative pain management; 2) increasing and significant neurological deficit; and 3) progressive cervical myelopathy. In most, persistence of pain is the primary indication. In this study except for one patient who had evidence of compressive myelopathy the remaining eleven patients were taken up for surgery for failure of conservative management to alleviate their pain and radicular symptoms.

Currently, anterior cervical discectomy with fusion is the procedure of choice for cervical disc disease, when the disc is removed anteriorly to avoid disc space collapse, prevent painful and abnormal cervical motion and speed intervertebral fusion. The use of graft prevents collapse of the disc space and maintains adequate foraminal size ${ }^{1}$. Graft collapse and failure of fusion was found in three patients in this study. This could have been avoided by adding an instrumentation. However, this did not have any influence on the surgical outcome as assessed by the VAS and Cervical Oswestry Score.

Patients with MRI documented cervical disc disease with compression of nerve roots that correlates well with the clinical symptoms and signs should be the indication for surgical management after conservative measures have failed. This criterion was strictly followed in our study.

Anterior cervical discectomy and fusion with autologous bone graft is the standard treatment for more than fifty years ${ }^{[5]}$.
According to some literature Instrumented ACDF leads to lesser graft dislocations and graft collapse and higher fusion rates [6]. On the other hand, complication rates after Instrumented ACDF is somewhat higher than uninstrumented ACDF. Taking this concept into consideration it could be said that the occurrence of graft collapse and failure of fusion could have been avoided if instrumentation had been done. However, it should be noted that this had not adversely affected the clinical outcome

Patient satisfaction was measured using a VAS scale, where a score of 0 indicated that the patient was satisfied and a score of 10 was graded as not satisfied. In our study twelve patients (84\%) had good score with no or mild pain, able to lift normal weights, no headache, drive with no difficulty and no sleep disturbances. One patient (8\%) had fair score with moderate pain, slight headache, difficulty in lifting weights, driving car and mild disturbance of sleep. One patient (8\%) had poor score with fairly severe pain, cannot lift normal weights, cannot do usual work, frequent headaches, difficulty in concentrating and driving and moderate disturbance of sleep. He was also having depression since he could not return to his old job.

This study with fewer number of patients may not help us in coming to a conclusion on the merits and demerits of ACDF as a surgical option for CDD that has failed to respond to conservative treatment.

\section{Conclusion}

There is no clear consensus regarding the optimal surgical procedure for cervical disc disease. ACDF is an effective treatment in patients who failed adequate period of conservative treatment. ACDF appears to be feasible, safe and has a good clinical outcome. The advantages of instrumentation in ACDF has not yet been established.

\section{References}

1. Campbell's Operative Orthopaedics, twelfth edition, 1919-1923.

2. ANGEVINE PD, ARONS RR, MCCORMICK PC. National and regional rates and variation of cervical discectomy with and without anterior fusion.2003;28;931-9

3. Jacobs Wc, Anderson Pg, Limbeek J. Single or double level anterior interbody fusion techniques for cervical disc disease. 2004, 18. CD004958

4. Cloward RB. The anterior approach for removal of ruptured cervical discs. J Neurosurg. 1958; 15:602-16.

5. Robinson Ra, Smith Gw. Anterolateral cervical disc removal and interbody fusion for cervical disc syndrome. Bull Johs Hopkins Hospital. 1955; 96:223-4.

6. Fountas KN, Kapsalaki EZ, Nikolakakos LG, Smisson $\mathrm{HF}$, Johnston KW, Grigorian AA et al. Anterior cervical discecyomy and fusion associated complications,Spine 2007; 32:2310-2317. 